ORIGINAL ARTICLE

\title{
Medication safety program reduces adverse drug events in a community hospital
}

\author{
M M Cohen, N L Kimmel, M K Benage, M J Cox, N Sanders, D Spence, J Chen
}

Qual Saf Health Care 2005;14:169-174. doi: 10.1136/qshc.2004.010942

See end of article for authors' affiliations

.....................

Correspondence to: Dr M M Cohen, Chief Medical Officer, NYU Hospitals Center, 550 First Avenue, HCC 1514, New York, NY 10016, USA; max.cohen@med.nyu.edu

Accepted for publication 13 December 2004

\begin{abstract}
Background: There is widespread interest in improving medication safety, particularly in the hospital setting. Numerous suggestions have been made as to how this should be done, but there is a paucity of data demonstrating the effectiveness of any of the interventions that have been proposed.

Objectives: To assess the impact of a wide ranging, community hospital based patient safety program on patient harm as measured by the rate of adverse drug events.

Design: An audit of discharged hospital patients was conducted from January 2001 to December 2003. Baseline data were collected for the first 6 months and multiple drug protocols and other interventions were instituted on the nursing units and in the pharmacy department over the subsequent 9 months (transition period). These interventions were largely based on information about medication risks acquired from internal medication event reporting. Each month of the study adverse drug events (ADE) were sought from a random sample of inpatient charts. A trigger tool was used to detect clues to ADEs, the presence of which was confirmed or excluded by detailed manual chart review. The severity of these events was categorized using the classification system of the National Coordinating Council for Medication Error and Reporting and Prevention.

Main outcome measures and results: Median ADEs per 1000 doses of medication dispensed declined significantly from 2.04 to 0.65 ( $p<0.001$ ). Median ADEs per 100 patient days declined significantly from 5.07 to 1.30 ( $p<0.001$ ). The proportion of inpatients with one or more ADE in the baseline period was $31 \%$ and declined threefold $(p<0.001)$. The severity of reported medication events also declined. The number of ADEs associated conclusively with patient harm was 1.67 per total doses delivered in the baseline period and declined eightfold $(p<0.001)$.

Conclusion: The implementation of a carefully planned series of low cost interventions focused on high risk medications, driven by information largely from internal event reporting, and designed to improve a hospital's medication safety leads to a significant decrease in patient harm.
\end{abstract}

\begin{abstract}
A wareness and scrutiny of medical errors, particularly in the hospital setting, has increased substantially in recent years. Attention has focused on medication errors because these represent the largest single component of all medical errors and many are life threatening. ${ }^{1}$ A wide range of relatively simple steps has been proposed that hospitals can take to reduce the likelihood of medication errors, ${ }^{2-4}$ but these interventions-while common sense and intrinsically sound-have not been subjected to formal study and there are few data to support their effectiveness. Seventy nine suggested safety practices were recently reviewed ${ }^{5}$ and 11 of these were highly rated for the weight of evidence in their support. Of these 11, only one was specifically designed to reduce the likelihood of a medication error.

More sophisticated and substantially more expensive solutions to the problem of medication errors-such as computer assisted management, ${ }^{6}$ computer based alerts, ${ }^{7}$ computerized physician order entry, ${ }^{8}$ advanced monitoring, ${ }^{9}$ bar coding ${ }^{10}$ and robotics ${ }^{11}$ - have been proposed, but their impact on the incidence of adverse drug events has not been consistently and reliably documented. ${ }^{12}{ }^{13}$

At Missouri Baptist Medical Center we launched our patient safety program by first addressing the culture of our organization and changing it from a punitive one to a just and fair one. We hired a full time patient safety specialist and, over a period of about a year among a broad menu of interventions, established executive safety rounds, an educational program for the entire staff, anonymous and simplified reporting of errors and events, feedback concerning interventions, and a rewards program for safety ideas. The success
\end{abstract}

of our patient safety program, as measured primarily by the rate of error reporting, has been previously reported. ${ }^{14}$ Armed with detailed knowledge of the medication errors that were being made, we were able to prioritize the most appropriate (based on frequency and risk) and potentially most cost effective corrective interventions. We then instituted a broad program of practical, low cost measures, including evidence based protocols, to improve medication safety at our hospital. These were largely directed at high risk medications. Concurrently, a failure modes and effects analysis (FMEA) ${ }^{15}$ was conducted of the pharmacy dispensing system to prevent dispensing errors before they occurred.

The primary purpose of this paper is to describe the impact of the medication safety component of our overall patient safety program on the harm caused to patients by medication errors.

\section{METHODS \\ Setting}

An audit of adverse drug events (ADE) was performed at Missouri Baptist Medical Center (MBMC) in St Louis, Missouri. MBMC is a not-for-profit 489 bed non-teaching suburban community hospital which is part of BJC HealthCare, a 13 hospital integrated healthcare delivery system.

\section{Design of study}

The institutional review board provided a waiver of this study. This audit was performed from January 2001 until 
December 2003. The study was divided into three time periods: baseline period from January 2001 until June 2001, transition period from July 2001 until March 2002, and postintervention period from April 2002 until December 2003. The transition period was defined as starting with the appointment of the hospital's full time patient safety specialist and lasting 9 months during which most of the key interventions were put in place. The dependent variables were ADEs per 1000 doses dispensed and ADEs per 100 patient days.

\section{Intervention}

Structural changes were made: a patient safety council was formed in May 2001, a full time patient safety specialist was hired in July 2001, and new event reporting systems were put in place. ${ }^{16}$ Most interventions were based on the information generated by these event reporting systems. Medication error reports originated from a variety of sources. These have been previously described ${ }^{14}$ and are listed in box 1 .

As a result of intensive work on culture change, medical error reporting increased significantly $(\mathrm{p}<0.001)$ from 35 per 1000 patient days in 2001 to 132 per 1000 patient days in 2003. ${ }^{14}$ All errors were entered into a single database. Reports run from the database identified high risk medications (such as insulin, narcotics, anticoagulants and antibiotics). Reports also identified which process step most frequently failed (prescribing, transcription, dispensing, administering, or monitoring). We were thus able to identify those drugs and/or processes that were most frequently associated with reported events. While event reporting encompassed all medical errors, this paper reports exclusively on medication errors and includes errors occurring at any step in the medication delivery system.

This resulted in the introduction of a number of drug protocols as shown in box 2. These protocols are available from the authors.

A variety of additional interventions were instituted based on the recommendations of the Institute for Safe Medication Practices (ISMP), the American Society of Health System Pharmacists (ASHP), the Institute for Healthcare Improvement (IHI), the Joint Commission for the Accreditation of Healthcare Organizations (JCAHO), and the Agency for Healthcare Research and Quality (AHRQ). These are shown in box 3.

Ten to twenty randomly (using a table of random numbers) selected charts of discharged inpatients were reviewed monthly from January 2001 until December 2003 to assess patient harm caused by medication errors. No patient was sampled more than once. Each chart was audited by two reviewers (a clinical pharmacist and a nurse manager) using an ADE trigger tool designed and tested by the IHI. ${ }^{17}$ An ADE was defined using the WHO definition: "a response to a drug which is noxious and unintended and which occurs at doses normally used in man for prophylaxis, diagnosis or therapy of disease, or the modification of physiological

\section{Box 1 Sources of error reports}

- Traditional written incident reports

- Medication event check box reporting form

- Pharmacy staff written reports

- Hotline calls

- Executive rounds

- Safety briefings on nursing units

- Direct calls to patient safety specialist

\section{Box 2 Protocols to improve medication safety}

- Weight based heparin

- Warfarin

- Lepirudin (Refludin) dosing

- Sedation

- Intravenous potassium

- Intravenous phosphate

- Sliding scale insulin

- Hypoglycemia

- Venous thromboembolism prophylaxis screening

- Clinical pharmacokinetics

- Enteral nutrition

- Total parenteral nutrition

Box 3 Other interventions to improve medication safety

- Drotrecogin Alfa (Xigris) prescribing and dosing protocol

- Standardized PCA orders

- Standardized postoperative nausea and vomiting orders

- Antibiotic conversion from IV to PO protocol

- Sub-acute rehabilitation weekly medication profile audits

function". Medication errors include more events than ADEs as they fail to account for unintended effects of drugs given appropriately. ADEs include any and all results that place patients at risk or expose them to harm. This instrument employs 24 triggers or clues suggestive of patient harm. If, on initial review, a trigger was identified by the reviewers, a more detailed review of the chart was performed to determine if there was an ADE that could reasonably be attributed to a medication error. If the reviewers reached different conclusions, this was resolved by obtaining the opinion of a critical care physician. Rozich et a l $^{17}$ used a sample size of 10 charts. This same sample size was used no matter the size of the hospital being studied. They found that increasing the sample size higher than 20 did not improve the reproducibility or reduce the variability of the data (personal communication). The majority of our monthly samples comprised 20 charts but on several occasions during the latter part of 2001 only 10 charts were reviewed because of staffing problems. The sample size was not based on a fixed percentage of total patients. The hospital has approximately 1700 discharges per month. Harm was defined as temporary or permanent impairment of physical or psychological body function or structure and includes transfers to a higher level of care or admission to a hospital as a result of the harm. The severity of harm of every ADE was scored using categories E to I of the National Coordinating Council for Medication Error Reporting and Prevention (NCCMERP) severity scoring $\operatorname{scale}^{18}$ (table 1).

These chart reviews for ADE, while designed as the main outcome measure of the impact of the medication safety program, also identified additional opportunities for improvement in the use of insulin (trigger: serum glucose less than $50 \mathrm{mg} / \mathrm{dl}$ ) and in narcotic management (trigger: oversedation, lethargy or fall). These opportunities were consistent 


\begin{tabular}{|c|c|}
\hline \multicolumn{2}{|c|}{$\begin{array}{l}\text { Table } 1 \text { NCCMERP index for categorizing } \\
\text { medication errors }\end{array}$} \\
\hline Category A & $\begin{array}{l}\text { Circumstances or events that have the } \\
\text { capacity to cause error }\end{array}$ \\
\hline Category B & $\begin{array}{l}\text { An error occurred but the error did not } \\
\text { reach the patient (an "error of omission" } \\
\text { does reach the patient) }\end{array}$ \\
\hline Category C & $\begin{array}{l}\text { An error occurred that reached the patient } \\
\text { but did not cause patient harm }\end{array}$ \\
\hline Category D & $\begin{array}{l}\text { An error occurred that reached the patient } \\
\text { and required monitoring to confirm that it } \\
\text { resulted in no harm to the patient and/or } \\
\text { required intervention to preclude harm }\end{array}$ \\
\hline Category $\mathrm{E}$ & $\begin{array}{l}\text { An error occurred that may have contributed } \\
\text { to or resulted in temporary harm to the } \\
\text { patient and required intervention }\end{array}$ \\
\hline Category $\mathrm{F}$ & $\begin{array}{l}\text { An error occurred that may have contributed } \\
\text { to or resulted in temporary harm to the } \\
\text { patient and required initial or prolonged } \\
\text { hospitalization }\end{array}$ \\
\hline Category G & $\begin{array}{l}\text { An error occurred that may have contributed } \\
\text { to or resulted in permanent patient harm }\end{array}$ \\
\hline Category $\mathrm{H}$ & $\begin{array}{l}\text { An error occurred that required intervention } \\
\text { necessary to sustain life }\end{array}$ \\
\hline Category I & $\begin{array}{l}\text { An error occurred that may have contributed } \\
\text { to or resulted in the patient's death }\end{array}$ \\
\hline
\end{tabular}

with the process deficiencies that were identified by the evaluation of reported errors. Adverse drug reactions were excluded from the study.

FMEA $^{15}$ conducted on the pharmacy dispensing system revealed a substantial number of opportunities for improving the safety of medication dispensing and all of these were instituted (box 4). Details of these interventions are available from the authors.

\section{Box 4 Interventions implemented as result of} FMEA of the medication dispensing system

- Decrease in not using the patient's profile to obtain medication from automated dispensing cabinet.

- Nursing units provided with larger refrigerators equipped with separate sections for each patient.

- Pharmacy staff picked up discontinued IVs and drugs three times daily.

- Safety checklist created to verify correct storage of IV fluids.

- Intravenous medication times printed on medication administration record (MAR).

- TALL-man multi colored lettering used when appropriate on medication packaging and on shelves in pharmacy.

- Installation of bar coded dispensing process for the automated dispensing cabinets (Pyxis ParX).

- Separation of "sound-a-likes" in Pyxis drawers.

- All subcutaneous doses greater than $1 \mathrm{ml}$ drawn up by IV room in a single syringe.

- Use of color coded CADD pumps for patient controlled analgesia or epidural administration of narcotics.

- Dispensing of all first dose antibiotics in green bag for easy identification.

- Standardization of PCA concentrations.

- Epinephrine $1 \mathrm{mg} / \mathrm{ml}$ ampoules in a ziplock bag with label stating "not for IV use, subcutaneous use only".

\section{Data analysis}

The rate of ADEs per 1000 doses dispensed by the hospital pharmacy and the rate of ADEs per 100 hospital days during the three time periods (baseline, transition and postintervention) were compared using the non-parametric Kruskal-Wallis test. The numbers of doses dispensed were the total number of medication doses dispensed to the patients randomly selected each month. These data were obtained from the pharmacy management system. The numbers of hospital days were the total number of patient days for the randomly selected patients each month. These data were obtained from the hospital's health information management department.

The change in the proportion of ADEs per 1000 doses dispensed by the pharmacy or 100 hospital days, and the change in the proportion of patients with an ADE during hospitalization in the three time periods were compared using the $\chi^{2}$ test for trend. Relative risks and $95 \%$ confidence intervals were calculated using EpiInfo Version 6. All other statistical tests were performed using SPSS 12.0 (SPSS Inc, Chicago, IL).

All data were tracked using statistical process control charts using three standard deviations to set the upper and lower control limits. The first center line value was selected in December 2001 using the first 11 points available. This gave a mean value of 1.79 ADE per 1000 doses and an upper control limit of 4.82. By July 2002 there were eight points on one side of the center line (Rule \#2 in Nelson's test) and at that point a new center line was calculated as $0.69 \mathrm{ADE}$ per 1000 doses with an upper control limit of 2.36. These was no special cause variation from that time through December 2003.

\section{RESULTS}

\section{Adverse drug events (ADEs)}

Examples of triggers identified and the associated ADE found on chart review include the following:

- A patient on coumadin with INR greater than 5 (trigger) subsequently developed a gastrointestinal bleed (ADE).

- A patient receiving two oral hypoglycemic medications and with severe hypoglycemia (trigger) subsequently required transfer to ICU (ADE).

- A patient in whom visual disturbance was noted (trigger), found to have a digoxin level twice the upper end of therapeutic range and an active order for digoxin (ADE).

The median ADE rates per 1000 doses delivered (interquartile range) were 2.04 (1.79-2.70) in the baseline period, $1.26(0.21-1.53)$ in the transition period, and $0.65(0.41-0.87)$ in the post-intervention period $(p=0.001)$. Comparison of the proportion of ADEs per total number of doses delivered in the three time periods showed a 3.6-fold lower risk of ADEs during the post-intervention period $\left(\mathrm{p}<0.001, \chi^{2}=30.253\right.$, table 2). The statistical process control chart (fig 1 ) for these data illustrates the time sequence of the reduction in ADE.

The median (interquartile range) ADE rates per 100 patient days were 5.07 (3.79-6.02) in the baseline period, 3.19 (0.58$5.03)$ in the transition period, and $1.30(0.87-1.71)$ in the post-intervention period $(p=0.001)$. Comparison of the proportions of $\mathrm{ADE}$ in the three time periods per 100 patient days also showed a 3.7-fold reduction in risk of ADE during the post-intervention period $\left(\mathrm{p}<0.001, \chi^{2}=34.115\right.$, table 2$)$.

The proportion of patients with ADEs in the baseline period $(31 \%)$ showed a 3.0 -fold reduction in risk of an ADE in the post-intervention period $\left(\mathrm{p}<0.001, \chi^{2}=25.000\right.$, table 2$)$.

\section{Severity of events}

The number of ADEs associated conclusively with patient harm (rated F-I) was 1.67 per total doses delivered in the 
Table 2 ADE rates for baseline, transition, and post-intervention period

\begin{tabular}{|c|c|c|c|c|c|}
\hline & Baseline & Transition & Post-intervention & $p$ value & $\chi^{2}$ for trend \\
\hline $\begin{array}{l}\text { Median (IQR) ADEs per } \\
1000 \text { doses dispensed }\end{array}$ & $2.04(1.79-2.70)$ & $1.26(0.21-1.53)$ & $0.65(0.41-0.87)$ & 0.001 & \\
\hline $\begin{array}{l}\text { Median (IQR) ADEs per } \\
100 \text { patient days }\end{array}$ & $5.07(3.79-6.02)$ & $3.19(0.58-5.03)$ & $1.30(0.87-1.71)$ & 0.001 & \\
\hline ADEs per total doses delivered & $36 / 16829$ doses & $18 / 15586$ doses & $34 / 55988$ doses & & \\
\hline RR for ADE (95\% CI) & 1.00 & $0.53(0.30$ to 0.94$)$ & 0.28 (0.18 to 0.45$)$ & $<0.001$ & 30.3 \\
\hline $\begin{array}{l}\text { ADEs per total number of } \\
\text { patient days }\end{array}$ & $36 / 730$ days & 18/593 days & $34 / 2604$ days & & \\
\hline RR for $A D E(95 \% \mathrm{Cl})$ & 1.00 & $0.63(0.36$ to 1.09$)$ & $0.27(0.17$ to 0.44$)$ & $<0.001$ & 34.1 \\
\hline $\begin{array}{l}\text { ADE severity F-I per total doses } \\
\text { delivered }\end{array}$ & 10 & 7 & 4 & & \\
\hline RR for $\operatorname{ADE}(95 \% \mathrm{Cl})$ & 1.00 & $0.74(0.28$ to 1.95$)$ & 0.12 (0.04 to 0.38$)$ & $<0.001$ & 17.7 \\
\hline $\begin{array}{l}\text { Patients with ADEs per total } \\
\text { number of charts reviewed* }\end{array}$ & $32 / 120$ charts & $16 / 90$ charts & $33 / 370$ charts & & \\
\hline RR for ADE $(95 \% \mathrm{Cl})$ & 1.00 & $0.67(0.39$ to 1.14$)$ & $0.33(0.22$ to 0.52$)$ & $<0.001$ & 25.0 \\
\hline
\end{tabular}

$A D E$, adverse drug event; $I Q R$, interquartile range; $R R$, relative risk compared with baseline; $\mathrm{Cl}$, confidence interval.

*Only one chart per patient was used.

baseline period and declined eightfold in the post-intervention period $\left(\mathrm{p}<0.001, \chi^{2}=17.734\right.$, table 2$)$. No patient deaths attributable to medication error were detected by the review of patient charts during this study. There were two life threatening events detected during the baseline period but none during the transition or post-intervention periods.

\section{Estimate of cost savings}

We estimate that, in our hospital, 4400 ADEs are now prevented each year as a result of our medication safety program. This is based on a reduction in the proportion of inpatients (total around 21000 per annum) experiencing an $\mathrm{ADE}$ from $30 \%$ to $10 \%$. Using the lowest published cost estimate and without adjusting to 2004 costs, this represents an annual cost saving of approximately $\$ 10000000$.

\section{Failure modes and effects analysis (FMEA)}

This resulted in a $69 \%$ reduction in the risk priority number for the pharmacy dispensing system over a period of 30 months.

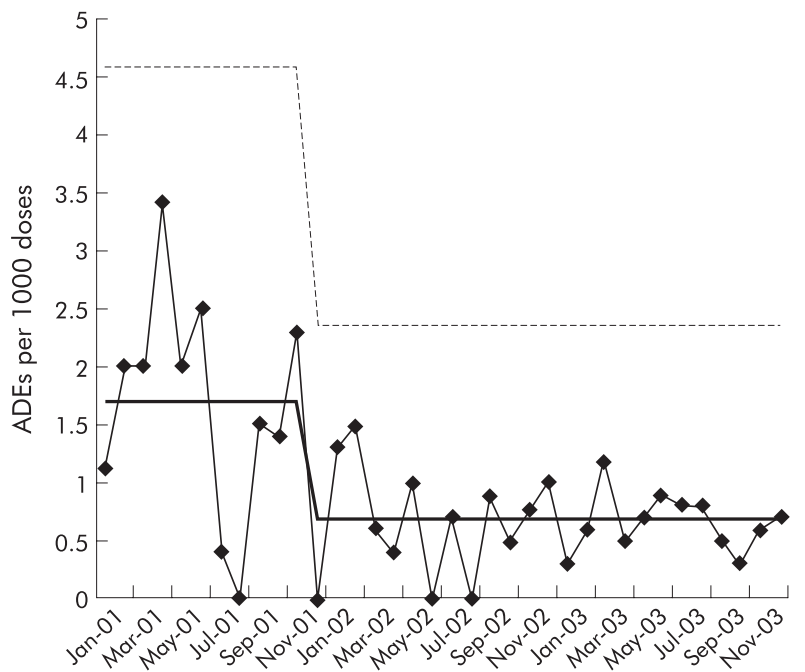

Figure 1 Statistical process control chart for adverse drug events (ADEs) per 1000 doses of medication dispensed during the entire period of audit from January 2001 until December 2003. The solid line represents the mean $A D E$ rate and the dotted line represents the upper control limit, defined as three standard deviations above the mean. Each point represents the result of a singe month's audit.

\section{DISCUSSION}

We have shown that a medication safety program comprising simple, common sense measures targeted by the findings of a rich adverse event reporting system will produce a significant and lasting reduction in patient harm as measured by ADEs. The major strength of our program is that it was inexpensive to implement compared with computerization and automation and almost certainly saved lives.

This program could not have been implemented without a full time dedicated patient safety specialist (NLK) whose knowledge of pharmaceuticals and whose commitment were crucial. It also required strong and passionate leadership and endorsement by the hospital's senior executives. ${ }^{16}$ The creation of a just and fair culture that encouraged and substantially increased event reporting ${ }^{14}$ was essential to its success. It also required that a significant number of hospital staff was encouraged to and actively participated in the work of a variety of medication safety teams. We have not attempted to quantify the cost of this management commitment to free staff from their regular duties.

There is currently a great deal of interest in patient safety and, in particular, medication safety, largely prompted by the seminal report in 1999 of the Institute of Medicine. ${ }^{19}$ Numerous suggestions have been made as to how hospitals can make medication ordering, transcription, dispensing, administration, and monitoring safer. Several organizations including the federal government have promoted specific recommendations $\mathrm{s}^{20-24}$ and business coalitions such as the Leapfrog Group ${ }^{25}$ have advocated strongly for computerized physician order entry (CPOE). Bates and Gawande, ${ }^{26}$ with an extensive experience of $\mathrm{CPOE}$, point out that, while it clearly prevents errors, it is expensive, is not readily available in an immediately usable form, and has not been shown to reduce patient harm. Nor has it been shown to be cost effective. In an accompanying editorial ${ }^{27}$ Berwick notes that, while the literature is replete with good ideas for improving patient safety, there is no evidence that these have actually made health care any safer.

We are committed to continuously improving medication safety and intend to implement CPOE and bar code scanning technology in due course. We considered, however, that the interventions reported here took priority over such expensive and problem prone programs, and that they were also essential to adequately prepare our hospital systems and the mind set of our medical staff and employees before embarking on these high technology initiatives.

The major limitations of this study are its reliance on the review of a sample of patient records, and that the detailed chart review, although conducted by two independent 
observers, was somewhat subjective in its determination of harm. The sample size was arbitrary and, while in most months comprised 20 charts, in several months in late 2001 comprised only 10 charts due to staffing problems. This may explain the large variation in the ADE rate seen during the last 6 months of 2001 (fig 1). There was, however, almost complete agreement between the two observers and between the conclusions they reached and that of an independent audit of 40 of the charts performed by IHI staff in August 2003. We are confident that our assessment of harm was accurate. Further, the median baseline rate of ADEs detected by us (2.04 per 1000 doses) was similar to the average rate detected among 30 community hospitals (2.61 per 1000 doses) by Rozich et al ${ }^{17}$ using the identical trigger tool. We know of no more accurate or objective method of detecting medication related harm to patients short of detailed manual review of every patient record. We consider that the results that we have obtained accurately reflect our entire inpatient population.

Another limitation is that we were unable to link any specific component of the medication safety program to a particular reduction in $\mathrm{ADE}$. It is not even possible to be certain which components of the intervention were most effective. We believe, however, that the most effective components were the pharmacy FMEA and the institution and widespread adoption of protocols for high alert medications. While this study was conducted at only one hospital, raising concerns about its generalizability, we believe that the interventions that we have implemented should be applicable to even the smallest hospital and that all of them could be adopted successfully.

The importance of having physician participation in these activities cannot be overestimated. A medication safety program is not likely to be successful if it is perceived to be designed and driven by either the pharmacy or the nursing staff acting alone. From its inception our medication safety committee was led by the medical director of critical care/ associate chief of the department of medicine (MJC). It reported regularly to the physician led Pharmacy and Therapeutics Committee. The patient safety council, to which it also reported, is the principal developer of patient safety policies and is chaired by the Chief Medical Officer (MMC). Physician opinion leaders were recruited to participate as members of every team that designed and/or implemented interventions such as new protocols. This generated hospitalwide acceptance of each initiative. These latter physicians served without financial compensation.

Another success factor was the method that we used to generate enthusiasm about and knowledge of our progress among hospital staff. Each month an electronic newsletter

\section{Key messages}

- There are few if any published data demonstrating that patient harm due to adverse drug events can be reduced by hospital based patient safety programs

- While most hospitals track medical errors, few make any attempt to measure actual harm.

- A hospital can substantially reduce the harm caused to patients from medication errors without going to the expense of computerized physician order entry.

- This improvement is gained primarily by changing the culture of safety to increase event reporting, then investigating the common causes of error, particularly those associated with high risk drugs. was distributed to everyone on the hospital internal email system that described the specific improvements that had been made to patient safety that month. This newsletter entitled "We heard you - We acted" was also posted where it could be read by all staff members and by patients and their families. This mechanism for providing feedback also served to help maintain error reporting and promote interest in the program.

The medication safety program has successfully reduced both the rate and severity of ADEs as detected by meticulous manual chart review. Most of these ADEs were preventable. Study of the severity of ADEs suggests that lives are probably being saved as a result of our medication safety program. The cost of preventable ADEs has been reported as ranging from $\$ 2500$ to $\$ 5000$ per case. ${ }^{28} 29$ The FDA, in its proposed rule for medication bar coding, ${ }^{30}$ estimated the cost of an ADE at \$2257. Our hospital's estimated cost savings of 10 million dollars per annum excludes all of the indirect organizational costs such as litigation costs, marketing costs, and the additional costs of hospital operations. ${ }^{31}$

\section{ACKNOWLEDGEMENTS}

The authors are grateful to the staff of the Institute for Healthcare Improvement and the hospital participants in the patient safety collaborative for freely sharing their ideas on patient safety with us, and to Margie Olsen PhD, MPH for statistical consultation.

\section{Authors' affiliations}

M M Cohen, N L Kimmel, M K Benage, M J Cox, N Sanders, D Spence, J Chen, Missouri Baptist Medical Center, BJC HealthCare, St Louis, Missouri, USA

\section{REFERENCES}

1 Leape LL, Brennan TA, Laird N, et al. The nature of adverse events in hospitalized patients. Results of the Harvard Medical Practice Study II. N Engl J Med 1991;324:377-84.

2 Institute for Safe Medication Practices (ISMP). www.ismp.org (accessed 1 December 2004).

3 Massachusetts Coalition for the Prevention of Medical Errors. MHA best practice recommendations to reduce medication errors. Burlington: Massachusetts Coalition for the Prevention of Medical Errors, 2001. Available at http://www.mhalink.org (accessed 1 December 2004).

4 American Hospital Association. AHA initiative: improving medication safety. Chicago, IL: American Hospital Association, 2002. Available at http:// www.aha.org (accessed 1 December 2003).

5 Shojania KG, Duncan BW, McDonald KM, et al. Making health care safer: a critical analysis of patient safety practices, Evidence Report/ Technology Assessment No 43. AHRQ publication 01-E058. Rockville, MD: Agency for Healthcare Research and Quality, 2001.

6 Evans RS, Pestotnik SL, Classen DC, et al. A computer-assisted management program for antibiotics and other antiinfective agents. N Engl J Med 1998;338:232-8

7 McMullin ST, Reichley RM, Watson LA, et al. Impact of a web-based clinical information system on cisapride drug interactions and patient safety. Arch Intern Med 1999; 159:2077-82.

8 Bates DW, Teich JM, Lee J, et al. The impact of computerized physician order entry on medication errors prevention. J Am Med Inform Assoc 1999;6:313-21.

9 Celi LA, HassanE, Manquard C, et al. The elCU: it's not just telemedicine. Crit Care Med 2001;29(Suppl):N183-9.

10 Gebhart F. VA facility slashes drug errors via bar-coding. Drug Topics 1999;1:44.

11 Bazzoli F. Medication errors. Automation holds promise of prevention. Health Data Manag 1995;3:30-32, 34, 36-39.

12 Oren E, Shaffer ER, Guglielmo BJ. Impact of emerging technologies on medication errors and adverse drug events. Am J Health-Syst Pharm 2003:60: 1447-58.

13 Murray MD. Automated medication dispensing devices. In:Making health care safer:a critical analysis of patient safety practices. AHRQ, UCSF-Stanford University Evidence-based Practice Center, June 2001. http:// www.ahrq.gov/clinic/ptsafety/chap 1 1.htm (accessed 30 September 2004).

14 Cohen MM, Kimmel NL, Benage MK, et al. Implementation of a hospitalwide patient safety program. Jt Comm J Qual Safety 2004;30:424-31.

15 Stamatis DH. Failure mode and effects analysis: FMEA from theory to execution. Milwaukee, WI: ASQC Quality Press, 1995.

16 Cohen MM, Eustis MA, Gribbins RE. Changing the culture of patient safety: Leadership's role in health care quality improvement. It Comm J Qual Safety 2003;29:329-35 
17 Rozich JD, Haraden CR, Resar RK. Adverse drug event tool: a practical methodology for measuring medication related harm. Qual Saf Health Care 2003; 12:194-200.

18 National Coordinating Council for Medication Error Reporting and Prevention. http://www.mers-tm.net (accessed 1 December 2004).

19 Institute of Medicine: To err is human. Building a safer health system. Washington DC: National Academy Press, 2000

20 Quality Interagency Coordination Task Force. Doing what counts for patient safety: Federal actions to reduce medical errors and their impact. Washington, DC: US Department of Health and Human Services, 2000.

21 Kizer KW. The National Quality Forum seeks to improve health care. Acad Med 2000;75:320-1

22 Findlay S, ed. Reducing medical errors and improving patient safety - success stories from the front lines of medicine. Washington, DC: National Coalition on Healthcare and the Institute for Healthcare Improvement, 2000.

23 Cohen MR, ed. Medication errors. Washington, DC: American Pharmaceutical Association, 1999.
24 American Hospital Association. Improving medication safety. AHA Quality Advisory 7 December, 1999

25 The Leapfrog Group for Patient Safety. Rewarding higher standards. http:// www.leapfroggroup.org (accessed 1 December 2004).

26 Bates DW, Gawande AA. Improving safety with information technology. N Engl J Med 2003;348:2526-34.

27 Berwick DM. Errors today and errors tomorrow. N Engl J Med 2003;348:2570-2.

28 Classen DC, Pestotnik SL, Evans RS, et al. Adverse drug events in hospitalized patients. Excess length of stay, extra costs, and attributable mortality. JAMA 1997; 277:301-6.

29 Bates DW, Spell N, Cullen DJ, et al. The costs of adverse drug events in hospitalized patients. JAMA 1997;277:307-11.

30 Food, Drug Administration (FDA). Bar code label requirement for human drug products and blood: proposed rule. Fed Reg 2003;68:12499-534.

31 Weeks WB, Waldron J, Foster T, et al. The organizational costs of preventable medical errors. Jt Comm J Qual Improv 2001;27:533-9.

$\mathrm{ECHO}$

\section{Informative video raises patients' satisfaction with cataract operations}

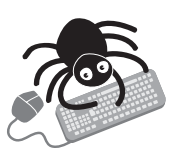

Please visit the Quality and

Safety in Health Care website [www.qshc. com] for a link to the full text of this article.
W de ranging, low cost benefits to patients, surgeons, and communities accrue from showing day case patients a video of what to expect from a cataract operation, claim Australian researchers, in a randomised controlled trial. Patients' satisfaction with the operation improved and anxiety lessened, regardless of the expected outcome or previous experience of the procedure.

Patients randomised to view a video about what the process would be like expected significantly higher levels of risk and pain than controls randomised to view a video about anatomy and development of cataract (mean score $2.48 v 1.6$ ), but after the operation they reported significantly more overall satisfaction $(8.19 v 7.84)$, better understanding $(7.44 v$ $5.82)$, and less anxiety $(0.88 v 1.29)$.

Patients with previous experience expected less anxiety and discomfort and the procedure was significantly closer to expectations, but viewing the expectations video still had significant effect. Interestingly, improvement occurred even though most- 84\%-of all patients declared before randomisation that they already had enough or too much information.

The trial included 141 patients in a private hospital carrying out the most private cataract operations in Sydney. Demographic data, details of past experience of the procedure and of expectations before and after the operations were obtained by a blinded interviewer, using a validated $12 \mathrm{~cm}$ visual analogue scale. Both videos were based on educational videos provided by pharmaceutical companies.

Using information to improve patients' expectations for cataract surgery has not been investigated much, even though this operation is the commonest performed in the private sector.

A Pager CK. British Journal of Ophthalmology 2005;89:10-13. 